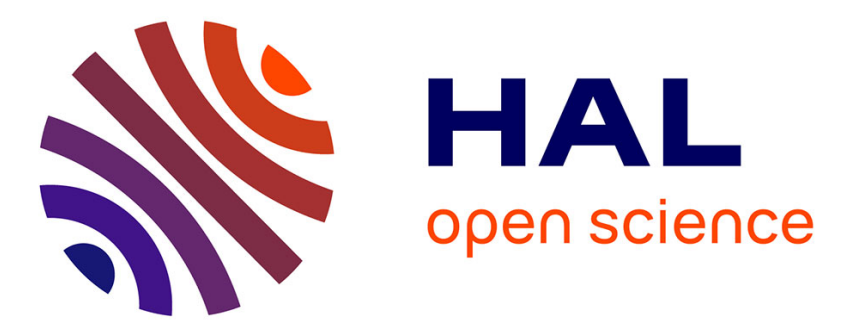

\title{
A general and efficient computational procedure for modelling the Kapitza thermal resistance based on XFEM
}

Julien Yvonnet, Qi-Chang He, Q.-Z. Zhu, J.-F. Shao

\section{- To cite this version:}

Julien Yvonnet, Qi-Chang He, Q.-Z. Zhu, J.-F. Shao. A general and efficient computational procedure for modelling the Kapitza thermal resistance based on XFEM. Computational Materials Science, 2011, 50 (4), pp.1220-1224. 10.1016/j.commatsci.2010.02.040 . hal-00559198

\section{HAL Id: hal-00559198 \\ https://hal.science/hal-00559198}

Submitted on 7 Sep 2014

HAL is a multi-disciplinary open access archive for the deposit and dissemination of scientific research documents, whether they are published or not. The documents may come from teaching and research institutions in France or abroad, or from public or private research centers.
L'archive ouverte pluridisciplinaire HAL, est destinée au dépôt et à la diffusion de documents scientifiques de niveau recherche, publiés ou non, émanant des établissements d'enseignement et de recherche français ou étrangers, des laboratoires publics ou privés. 


\title{
A general and efficient computational procedure for modelling the Kapitza thermal resistance based on XFEM
}

\author{
J.Yvonnet ${ }^{\mathrm{a}, *}$, Q.-C. He ${ }^{\mathrm{a}}$, Q.-Z. Zhu, ${ }^{\mathrm{b}}$ J.-F. Shao ${ }^{\mathrm{b}}$ \\ a Université Paris-Est, Laboratoire Modélisation et Simulation Multi Échelle, MSME UMR 8208 CNRS, 5 Bd Descartes, \\ F-77454 Marne-la-Vallée cedex, France \\ ${ }^{\mathrm{b}}$ Universite Lille 1, Laboratoire de Mecanique de Lille, UMR 8107 CNRS, Boulevard Paul Langevin, 59655 Villeneuve \\ d'Ascq Cedex, France
}

\begin{abstract}
The thermal resistance of an interface between two materials, conceptualized by Kapitza, is an important physical phenomenon encountered in many situations of practical interest. The numerical treatment of this phenomenon has up to now run into difficulties due to the temperature discontinuity. In this work, a general and efficient computational procedure for modelling the Kapitza thermal resistance is proposed, which is based on the extended finite element method (XFEM) in tandem with a level-set method. The steady thermal conduction in a two-phase material with the Kapitza thermal resistance at the interface is first formulated in a variational way and then numerically treated with the proposed computational procedure. Different three-dimensional numerical examples with known analytical solutions show the high accuracy and robustness of the proposed computational procedure in capturing the temperature jump across an interface.
\end{abstract}

Key words: Kapitza resistance; XFEM; Level-set; Imperfect interfaces

\section{Introduction}

An interface between two materials often exhibits a thermal resistance posing a barrier to heat flow and leading to a temperature jump across the interface. This phenomenon was observed and conceptualized by Kapitza [7] who measured the thermal interface resistance between various metals and liquid helium. The thermal resistance also occurs at solids/solids interfaces, as shown experimentally in many works (see, e.g., [4,16,14,3]). Accounting for the Kapitza thermal resistance is especially important to largescale integrated circuitry, components and sensors, because the heat transport and energy dissipation

\footnotetext{
* Correspondance to J.Yvonnet

Email address: julien.yvonnet@univ-paris-est.fr (J.Yvonnet).
}

through interfaces in such systems are comparable or even dominant over those in bulk. In materials science, the Kapitza thermal resistance has long been recognize to be of theoretical and practical importance (see, e.g., [11] [17]).

The numerical modelling of the Kapitza thermal resistance has up to now run into difficulties. Indeed, the treatment of the temperature jump at an interface requires a discontinuous temperature approximation, which cannot be handled with the classical Finite Element Method (FEM) or Boundary Element Method (BEM) [9]. In the context of FEM, the meshing of an interface is needed and has to conform with that of the neighbouring volume parts, and appropriate surface elements must be constructed [5]. For interfaces of complex geometry, this is a difficult issue. In the present work, we propose a general and efficient computational procedure for modelling the 
Kapitza thermal resistance, which is based on the extended finite element method (XFEM) $[1,10,15,2]$ in tandem with a level-set method [12]. The steady thermal conduction in a two-phase material with the Kapitza thermal resistance at an arbitrarily shaped interface is first formulated in a variational way and then numerically treated with the proposed computational procedure. In particular, the interface is described implicitly in a regular background mesh through a level-set method; the temperature jump is ensured by a discontinuous scheme based on enrichment functions. This method is simple and does not require any surface elements. Finally, we show the high accuracy and robustness of our computational procedure in modelling the Kapitza thermal resistance through benchmarks for which exact analytical solutions are available.

\section{Formulation of the problem}

\subsection{Strong form}

Let a body $\Omega$ be divided by an interface $\Gamma$ into two parts $\Omega^{(1)}$ and $\Omega^{(2)}$, possibly made of two different solid phases, and let $\mathbf{x}$ be the position vector of a current point in $\Omega$. The solid-solid interface $\Gamma$ is assumed to exhibit the Kapitza thermal resistance $\alpha$. Thus, the thermal behavior of $\Gamma$ is specified by

$$
\begin{gathered}
\llbracket q_{n} \rrbracket=0 \text { on } \Gamma, \\
\llbracket T(\mathbf{x}) \rrbracket=-\alpha q_{n} \text { on } \Gamma,
\end{gathered}
$$

where $\llbracket . \rrbracket=(.)^{(2)}-(.)^{(1)}$ denotes the jump across $\Gamma, q_{n}$ is the normal heat flux and $T$ the temperature. The Kapitza resistance $\alpha$ can be evaluated at different temperatures by measuring the ratio of the temperature drop to the heat flux across an interface [17]. Adopting the Fourier law for the bulk solid phases, the steady heat flux and temperature fields can be obtained by solving the problem formulated by the fields equations

$$
\begin{aligned}
& \nabla \cdot \mathbf{q}(\mathbf{x})-r(\mathbf{x})=0 \text { in } \Omega^{(i)}, \quad i=1,2, \\
& \mathbf{q}(\mathbf{x})=-\mathbf{k}^{(i)}(\mathbf{x}) \nabla T(\mathbf{x}), \quad i=1,2,
\end{aligned}
$$

together with the interface equations (1) and (2) and some appropriate boundary conditions. Above, $\mathbf{q}(\mathbf{x})$ denotes the heat flux, $r(\mathbf{x})$ is a heat source term and $\mathbf{k}^{(i)}$ the conductivity tensor of phase $i$. In a rather general way, we consider the Dirichlet and Neumann boundary conditions prescribed on the complementary and disjoints portions of $\partial \Omega$, denoted by $\partial \Omega_{T}$ and $\partial \Omega_{q}$, as follows:

$$
\left\{\begin{array}{l}
\mathbf{q} \cdot \boldsymbol{\nu}=-\bar{q}_{\nu} \text { on } \partial \Omega_{q}, \\
T=\bar{T} \text { on } \partial \Omega_{T} .
\end{array}\right.
$$

In the above, $\boldsymbol{\nu}$ is the outward unit vector normal to $\partial \Omega$. For later use and without loss of generality, let the interface $\Gamma$ be characterized by a function $\phi$ : $\mathbb{R}^{3} \rightarrow \mathbb{R}$ as its zero-level set:

$$
\Gamma=\left\{\mathbf{x} \in \mathbb{R}^{3} \mid \phi(\mathbf{x})=0\right\} .
$$

The unit vector $\mathbf{n}$ normal to $\Gamma$ is directed from $\Omega^{(1)}$ into $\Omega^{(2)}$

\subsection{Weak form}

For the sake of clarity, we assume $\partial \Omega_{q} \subseteq \partial \Omega^{(2)}$. The weak form associated with Eqs. (3)-(5) is given as follows:

Find $T^{(i)} \in \mathcal{D}=\left\{T=\bar{T}\right.$ on $\left.\partial \Omega_{T}^{(i)}, T \in H^{1}\left(\Omega^{(i)}\right)\right\}$ with $i=1$ or 2 , such that

$$
\begin{aligned}
& \int_{\Omega^{(1)}} \mathbf{q}^{(1)} \cdot \boldsymbol{\nabla}\left(\delta T^{(1)}\right) d \Omega-\int_{\Gamma} \mathbf{q}^{(1)} \cdot \mathbf{n} \delta T^{(1)} d \Gamma \\
& \quad+\int_{\Omega^{(1)}} r \delta T^{(1)} d \Omega=0 \\
& \int_{\Omega^{(2)}} \mathbf{q}^{(2)} \cdot \nabla\left(\delta T^{(2)}\right) d \Omega+\int_{\Gamma} \mathbf{q}^{(2)} \cdot \mathbf{n} \delta T^{(2)} d \Gamma \\
& \quad+\int_{\Omega^{(2)}} r \delta T^{(2)} d \Omega+\int_{\partial \Omega_{q}} \bar{q}_{\nu} \delta T^{(2)} d \Gamma=0,
\end{aligned}
$$

for all $\delta T^{(i)} \in H_{0}^{1}\left(\Omega^{(i)}\right)$ with

$$
H_{0}^{1}\left(\Omega^{(i)}\right)=\left\{\delta T \in H^{1}\left(\Omega^{(i)}\right), \delta T=0 \text { on } \partial \Omega_{T}^{(i)}\right\} .
$$

Adding Eqs. (7) and (8) yields:

$$
\begin{aligned}
\int_{\Omega} \mathbf{q} & \cdot \nabla(\delta T) d \Omega+\int_{\Gamma}\left(\mathbf{q}^{(2)} \cdot \mathbf{n} \delta T^{(2)}-\mathbf{q}^{(1)} \cdot \mathbf{n} \delta T^{(1)}\right) d \Gamma \\
& +\int_{\Omega} r \delta T d \Omega+\int_{\partial \Omega_{q}} \bar{q}_{\nu} \delta T d \Gamma=0 .
\end{aligned}
$$

Invoking the continuity of $q_{n}$ on $\Gamma$, we obtain

$$
\begin{gathered}
\int_{\Omega} \mathbf{q} \cdot \nabla(\delta T) d \Omega+\int_{\Gamma} q_{n} \llbracket \delta T \rrbracket d \Gamma \\
+\int_{\Omega} r \delta T d \Omega+\int_{\partial \Omega_{q}} \bar{q}_{\nu} \delta T d \Gamma=0 .
\end{gathered}
$$

Introducing (2) and (4) into the above expression leads finally to:

$$
\begin{aligned}
& \int_{\Omega} \mathbf{k}(\mathbf{x}) \nabla T \cdot \nabla(\delta T) d \Omega+\int_{\Gamma} \llbracket T \rrbracket \frac{1}{\alpha} \llbracket \delta T \rrbracket d \Gamma \\
& =\int_{\Omega} r \delta T d \Omega+\int_{\partial \Omega_{q}} \bar{q}_{\nu} \delta T d \Gamma .
\end{aligned}
$$


This equation is the basis for the following numerical treatment.

\section{Computational procedure based on XFEM and the level-set method}

The domain $\Omega$ is discretized by nodes that do not necessarily match the interface $\Gamma$. Here tetrahedra are adopted for meshes for the reason of simplicity and robustness. Regular meshes can then be chosen for parallelepipedic domains, even if the interface is arbitrarily shaped.

The interface $\Gamma$ being defined by (6), the unit normal vector $\mathbf{n}(\mathbf{x})$ can be numerically evaluated by

with

$$
\mathbf{n}(\mathbf{x})=\frac{\boldsymbol{\nabla} \phi(\mathbf{x})}{\|\boldsymbol{\nabla} \phi(\mathbf{x})\|}
$$

$$
\nabla_{i} \phi(\mathbf{x})=\sum_{j=1}^{n} \frac{\partial N_{j}(\mathbf{x})}{\partial x_{i}} \phi_{j},
$$

where $N_{j}(\mathbf{x})$ are standard finite element shape functions, $\phi_{j}$ are the nodal values of the level-set function $\phi, n$ is the number of nodes of an element containing $\mathbf{x}$. In the present paper, we employ linear finite element shape functions in tetrahedral elements.

The approximation of the temperature at a point $\mathbf{x}$ inside an element $\Omega_{e}$ is given by:

$$
T^{h}(\mathbf{x})=\sum_{i=1}^{n} N_{i}(\mathbf{x}) T_{i}+\sum_{j=1}^{m} N_{j}(\mathbf{x}) \Psi(\mathbf{x}) a_{j} .
$$

The second term in the right-hand member of this equation is intended for capturing the temperature jump across $\Gamma$. Precisely, $a_{j}$ are the additional unknowns, and $\Psi$ is the enrichment function which takes the form

$$
\Psi(\mathbf{x})=\frac{1}{2} \operatorname{sgn}\left(\sum_{i=1}^{n} N_{i}(\mathbf{x}) \phi_{i}\right) .
$$

In the second term of Eq. (12), the approximated temperature jump has the expression

$$
\llbracket T^{h}(\mathbf{x}) \rrbracket=T^{h}\left(\mathbf{x}^{+}\right)-T^{h}\left(\mathbf{x}^{-}\right),
$$

where the superscripts + and - denote the sides of $\Gamma$. Introducing (15) and (16) into (17) and invoking the continuity of $N_{i}(\mathbf{x})$ across $\Gamma$, we obtain

$$
\llbracket T^{h}(\mathbf{x}) \rrbracket=\sum_{j=1}^{m} N_{j}(\mathbf{x}) a_{j}
$$

The expressions of the approximated temperature variation $\delta T^{h}$ and jump $\llbracket \delta T^{h} \rrbracket$ are taken to be similar to those of $T^{h}$ and $\llbracket \delta T^{h} \rrbracket$.

With the aid of (15) and (18), the discrete approximation of (12) is finally provided by the system of linear equations

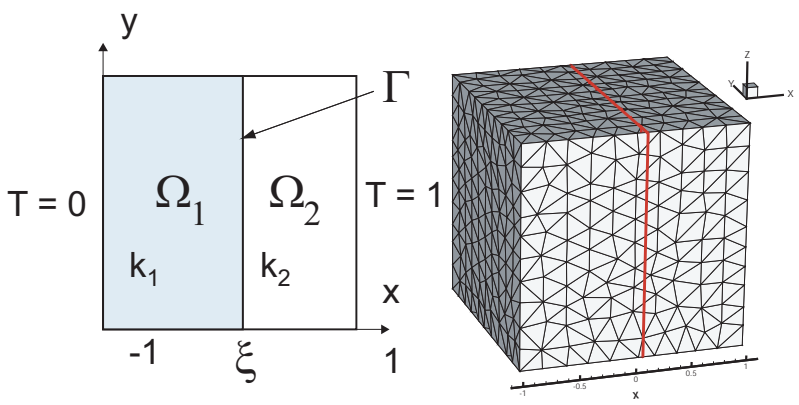

Fig. 1. Discontinuous patch test related to interfacial resistance: (a) Geometry; (b) Mesh and interface.

$$
\left(\mathbf{K}+\mathbf{K}^{s}\right) \mathbf{d}=\mathbf{Q} .
$$

The matrix $\mathbf{K}$, associated with the bulk thermal conductivity, is defined by

$$
\mathbf{K}=\int_{\Omega} \mathbf{B}^{T} \mathbf{k}(\mathbf{x}) \mathbf{B} d \Omega
$$

where $\mathbf{B}$ is the matrix of shape functions derivatives. In (19), the matrix $\mathbf{K}^{s}$, due to the Kapitza thermal resistance of $\Gamma$, is specified by

$$
\mathbf{K}^{s}=\int_{\Gamma} \frac{1}{\alpha} \tilde{\mathbf{N}}^{T} \tilde{\mathbf{N}} d \Gamma
$$

where $\tilde{\mathbf{N}}$ is the matrix defined through $\llbracket T^{h} \rrbracket=\tilde{\mathbf{N}} \mathbf{d}^{e}$ with $\mathbf{d}^{e}$ being the nodal unknowns vector of element $\Omega^{e}$. Finally, $\mathbf{Q}$ is the generalized heat flux vector calculated by

$$
\mathbf{Q}=\int_{\partial \Omega_{q}} \mathbf{N}^{T} \bar{q}_{\nu} d \Gamma+\int_{\Omega} \mathbf{N}^{T} r d \Omega
$$

where $\mathbf{N}$ is the matrix of shape functions and $\mathbf{d}$ is the vector of generalized unknowns.

The numerical integration of the volume integrals is carried out by employing one Gauss point in non enriched elements (not intersected by the interface). Enriched elements are subdivided into tetrahedra on each side of the interface $\Gamma$, and the four-point Gauss quadrature is applied to each sub-tetrahedron. For the surface integral, the interface is approximated by triangles resulting from the intersection of the zero-level-set and the tetrahedral mesh. The threepoints Gauss quadrature scheme is applied to the collection of triangles.

\section{Numerical examples}

\subsection{Discontinuous patch test related to interfacial resistance}

In this example, we propose a benchmark with a planar and resistive interface. The problem geome- 
Table 1. Relative energy error norm for the discontinuous patch test.

\begin{tabular}{cccc}
\hline$\alpha \backslash k_{1} / k_{2}$ & 1 & 10 & 100 \\
\hline 0 & $1.7 \times 10^{-8} 2.2 \times 10^{-8} 2.2 \times 10^{-8}$ \\
1 & $1.9 \times 10^{-8} 2.2 \times 10^{-8} 2.2 \times 10^{-8}$ \\
10 & $1.9 \times 10^{-8} 2.2 \times 10^{-8} 2.2 \times 10^{-8}$ \\
$10^{3}$ & $1.9 \times 10^{-8} 2.2 \times 10^{-8} 2.2 \times 10^{-8}$ \\
\hline
\end{tabular}

try is defined in figure 1. By considering equilibrium in each phase, boundary conditions $T(-1)=0$ and $T(1)=1$, the continuity of normal flux across the interface located at $(x=\xi)$ and the one-dimensional jump condition $\llbracket T(\xi) \rrbracket=-\alpha q_{n}(\xi)$, we obtain the following exact solution:

with

$$
\left\{\begin{array}{l}
T(x)=\frac{k_{2}(x+1)}{\gamma} \forall x \in[-1 ; \xi[ \\
\left.\left.T(x)=\frac{\left(k_{1} x+\beta\right)}{\gamma} \forall x \in\right] \xi ; 1\right]
\end{array}\right.
$$

$$
\left\{\begin{array}{l}
\gamma=\xi\left(k_{2}-k_{1}\right)+k_{1}\left(1+\alpha k_{2}\right)+k_{2} \\
\beta=\xi\left(k_{2}-k_{1}\right)+k_{2}\left(1+\alpha k_{1}\right)
\end{array}\right.
$$

Different computations are performed for different values of the resistance $\alpha$ ranging from $10^{-5}$ $\mathrm{W} / \mathrm{m}^{2} \mathrm{~K}$ (nearly perfect interface) to $1000 \mathrm{~W} / \mathrm{m}^{2} \mathrm{~K}$ (nearly insulating interface) and for different value of the ratio $k_{1} / k_{2}, k_{2}=1 \mathrm{~W} / \mathrm{mK}$. A unidirectional conduction model $\left(\mathbf{k}^{(i)}=k_{i} \mathbf{e}_{1} \otimes \mathbf{e}_{1}\right)$ is chosen to render the solution purely one-dimensional. The interface position is $\xi=0.09 \mathrm{~m}$. The mesh is not regular, with roughly 11 nodes on each sides of the cube.

The relative energy error norm (see e.g. [18]) is computed for different values of $k_{i}$ and various interface positions. The results obtained are reported in Table 1, indicating that the method allows passing this discontinuous patch test roughly at the machine precision, and the temperature discontinuity is accurately reproduced.

In figure 2 , the calculated and exact temperature fields are plotted versus the $x$ coordinate for $k_{1}=0.1$ $\mathrm{W} / \mathrm{mK}$ and $k_{2}=1 \mathrm{~W} / \mathrm{mK}$. We can observe that the strong temperature discontinuity is well captured by the proposed numerical procedure.

\subsection{Benchmark with spherical thermally resistive interface}

A composite sphere $\Omega$ is composed of a coating of outer radius $r_{2}$ and a core of radius $r_{1}$, which are separated by an interface with the Kapitza re-

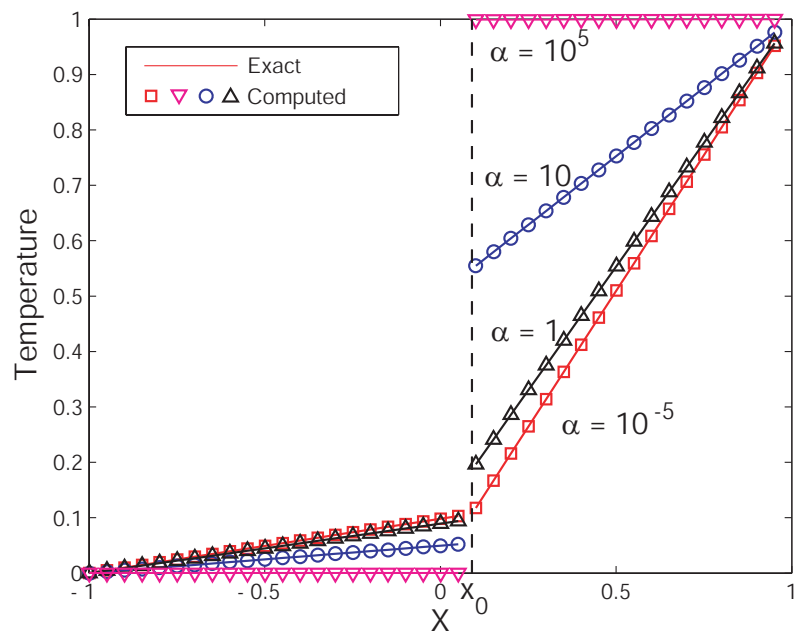

Fig. 2. Exact and computed solutions to the discontinuous patch test for different interface resistances $\alpha, k_{1}=0.1$ $\mathrm{W} / \mathrm{mK}$ and $k_{2}=1 \mathrm{~W} / \mathrm{mK}$.

sistance $\alpha$. This spherical composite is submitted to a homogeneous temperature gradient field

$$
T=\bar{T}=-\mathbf{e}^{0} \cdot \mathbf{x}
$$

on its boundary $\partial \Omega$. In $(25), \mathbf{e}_{0}$ is a constant vector and is, with no loss of generality, set to be $\mathbf{e}^{0}=$ $\left(0,0, e^{0}\right)^{T}$. Both the core and coating forming the composite sphere are assumed to be isotropic and individually homogeneous.

Then, the temperature and heat flux fields are, in the system of cordinates $(r, \theta, \varphi)$ associated to an orthonormal basis $\left(\mathbf{e}_{r}, \mathbf{e}_{\theta}, \mathbf{e}_{\varphi}\right)$, given by (see, e.g., [6])

$$
\begin{gathered}
T^{(i)}=-\left(a_{i} r+\frac{b_{i}}{r^{2}}\right) \cos \omega, \\
q_{r}^{(i)}=k^{(i)}\left(a_{i}-2 \frac{b_{i}}{r^{3}}\right) \cos \omega, \\
q_{\theta}^{(i)}=-k^{(i)}\left(a_{i}+\frac{b_{i}}{r^{3}}\right) \sin \omega, q_{\varphi}^{(i)}=0,
\end{gathered}
$$

In these expressions, $\omega$ denotes the angle between $\mathbf{e}^{0}$ and $\mathbf{e}_{r}, i=1$ refers to the core and $i=2$ is relative to the coating. By writing the continuity conditions at the interface $r=r_{1}$ and avoiding the singularity at $r=0$, it follows that

$$
\begin{aligned}
& a_{1}=\frac{3 k_{2} r_{1} r_{2}^{3}}{\beta+\gamma} e_{0}, a_{2}=\frac{\beta}{\beta+\gamma} e_{0} \\
& b_{2}=\frac{\gamma}{\beta+\gamma} e_{0} \\
& \beta=\left(k_{1} r_{1}+2 k_{2}\left(\alpha k_{1}+r_{1}\right)\right) r_{2}^{3} \\
& \gamma=\left(k_{1} k_{2} \alpha+\left(k_{2}-k_{1}\right) r_{1}\right) r_{1}^{3}
\end{aligned}
$$




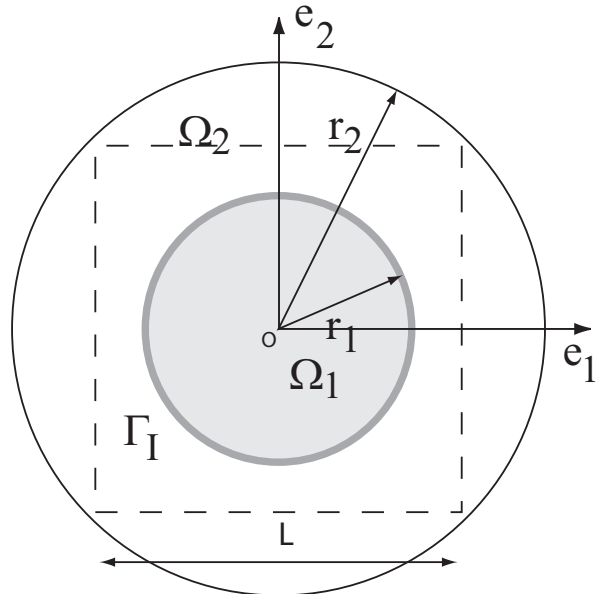

(a)

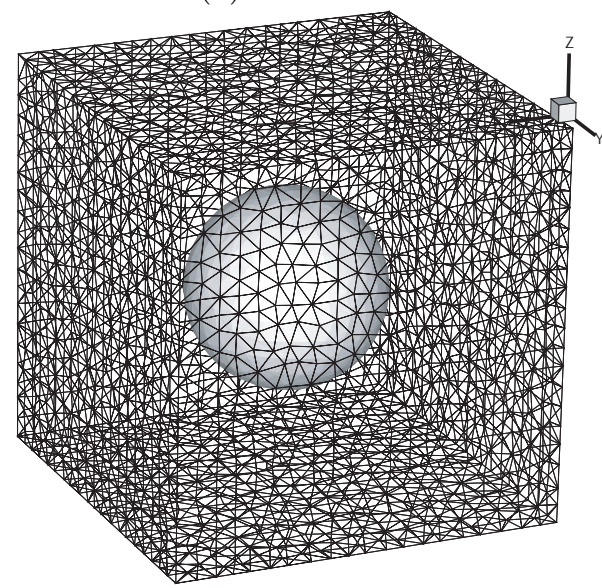

(b)

Fig. 3. Problem of a composite sphere: (a) geometry; (b) mesh of the cube cut out of the sphere.

We cut out a cubic domain of side length $L=4 r_{1}$ out of a sphere as shown in figure 3 , and prescribe the exact temperature solution (26) on its external boundary. The numerical parameters are $r_{1}=1$ $\mathrm{m}, r_{2}=3 \mathrm{~m}, k_{1}=0.01 \mathrm{~W} / \mathrm{mK}, k_{2}=1 \mathrm{~W} / \mathrm{mK}$, $e^{0}=1$. The convergence of the numerical solution is examined for different values of the Kapitza resistance $\alpha$ ranging from $\alpha=10^{-5} \mathrm{~W} / \mathrm{m}^{2} \mathrm{~K}$ to $\alpha=10^{3}$ $\mathrm{W} / \mathrm{m}^{2} \mathrm{~K}$. The corresponding results are presented in figure 4 . The convergence rate $R$ is in all cases close to the optimal one $R=1$.

The temperature field is plotted versus radius $r$ for $\alpha=10 \mathrm{~W} / \mathrm{K}$ and $\alpha=100 \mathrm{~W} / \mathrm{K}$ in figure 5 , along a line $\mathbf{x}=-r \mathbf{e}_{z}$. The latter shows that the temperature jump across the interface is accurately captured.

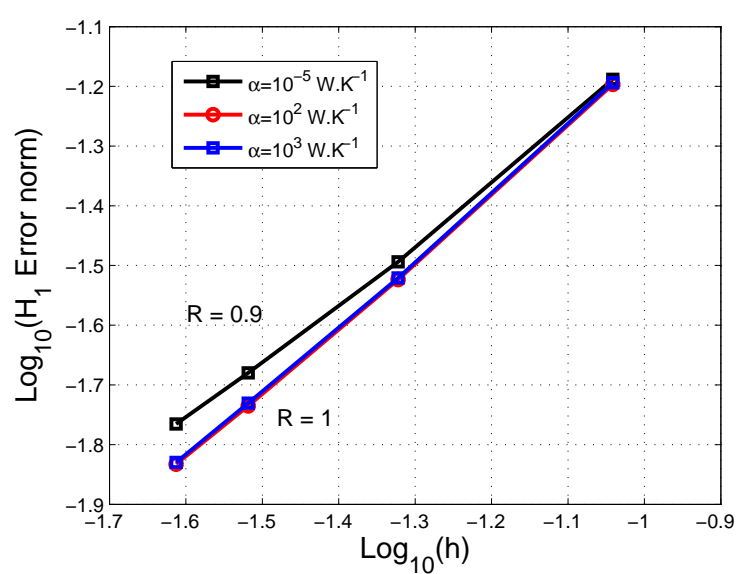

Fig. 4. Convergence analysis for the thermal problem of imperfectly bonded inclusion with different interfacial resistances

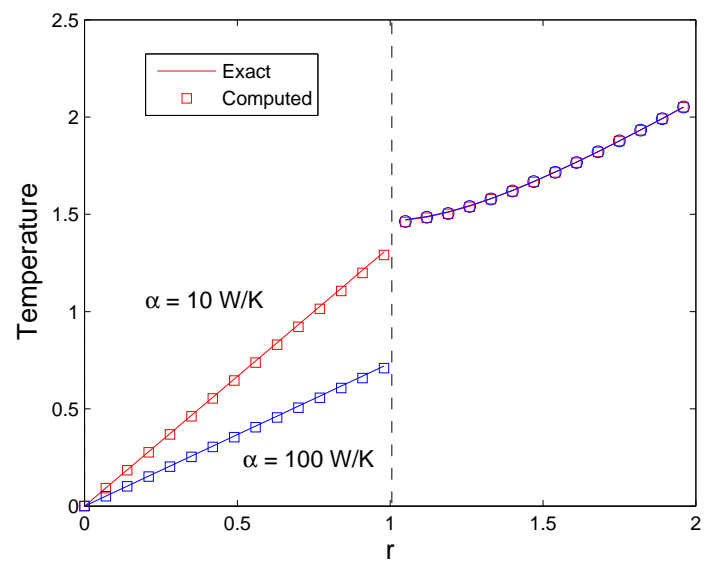

Fig. 5. Exact and computed solutions for the spherical benchmark problem for different values of the Kapitza resistance $\alpha$.

Finally, let us consider another problem in which both the bulk phases consists of the same material $\left(k_{1}=k_{2}=1 \mathrm{~W} / \mathrm{mK}\right)$, the Kapitza resistance varies between $\alpha=10^{-5} \mathrm{~W} / \mathrm{m}^{2} \mathrm{~K}$ (nearly perfectly conducting interface) and $\alpha=10^{3} \mathrm{~W} / \mathrm{m}^{2} \mathrm{~K}$ (nearly perfectly insulating interface), and the boundary conditions are the same as in the former problem. The computated temperature field is plotted in a plane tangent to $\mathbf{e}^{0}$ and passing by the origin in figure 6 . The influence of the Kapitza resistance on the continuity of the temperature field is clearly reflected even though the interface does not conform to the tetrahedral mesh. The observed oscillations are only due to the post-processing which does not take into account the discontinuity in the elements. 


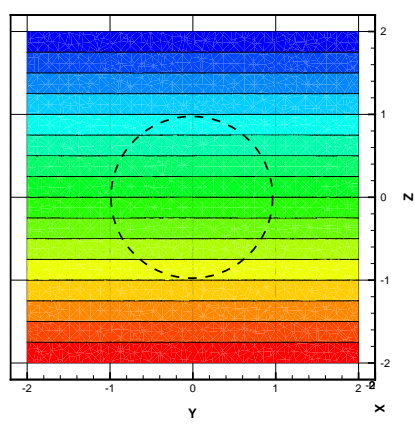

(a)

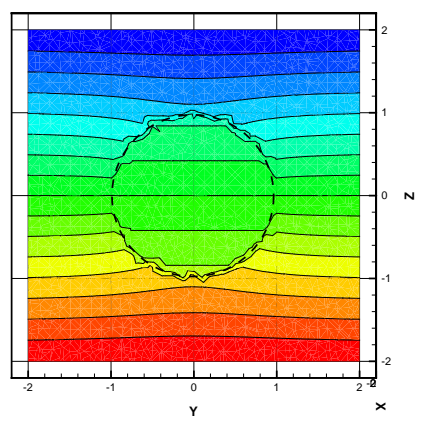

(b)

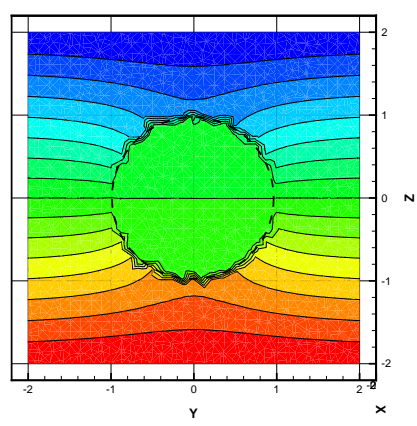

(c)

Fig. 6. Effect of the Kapitza resistance coefficient $\alpha$ on the temperature field: (a) $\alpha=0$; (b) $\alpha=1$; (c) (b) $\alpha=10^{3}$.

\section{Conclusion}

A simple and general numerical procedure has been proposed to model the Kapitza thermal resistance at an arbitrarily shaped interface. This procedure is framed within the extended finite element method combined with the level-set method. Its high accuracy and robustness have been evidenced by the benchmark tests presented. It can be used to study the effects of the Kapitza thermal resistance occuring not only in materials but also in many other systems such as large-scale integrated circuitry, device components and sensors.

\section{References}

[1] Belytschko T, Black T. Elastic crack growth in finite elements with minimal remeshing. Int. J. Numer. Methods Eng. 1999;45(5):601-620.

[2] Belytchko T, Parimi C, Moës N, Sukumar N, Usui S. Structured extended finite element method for solids defined by implicit surfaces. Int. J. Numer. Methods Eng. 2003;56:609-635.

[3] Cahill DG, Ford WK, Goodson KE, Mahan GD, Majumdar A, Maris HJ, Merlin R, Phillpot SR. Nanoscale thermal transport. J. Appl. Phys. 2003;93(2):793-818.

[4] Challis LJ, Kapitza resistance and acoustic transmission across buondaries at high-frequencies. J. Phys. C. 1974;7:481-495.

[5] Graham S., McDowell D.L. Numerical analysis of the transverse thermal conductivity of composites with imperfect interfaces. J. Heat Transfer. 2003;125:389-393.

[6] Hervé E. Thermal and thermoelastic behaviour of mutliply coated inclusion-reinforced composites. Int. J. Solids Struct. 2002;39:1041-1058.

[7] Kapitza PL. J. Phys. 1941;4:181.

A BEM analysis for thermomechanical closure of interfacial cracks incorporating friction and thermal resistance G.I. Giannopoulos, N.K. Anifantis

[8] Giannopoulos GI, Anifantis NK. A BEM analysis for thermomechanical closure of interfacial cracks incorporating friction and thermal resistance. Comput. Methods Appl. Mech. Engrg. 2007;196:1018-1029.

[9] Mahan GD. Kapitza thermal resistance between a metal and a nonmetal. Phys. Rev. B. 2009;79:075408.

[10] Moës N, Dolbow J, Belytschko T. A finite element method for crack growth without remeshing. Int. J. Numer. Methods Eng. 2009;46(1):131-156.

[11] Nan C.-W., Birringer R., Clarke D.R., Gleiter H. Effective thermal conductivity of particulate composites with interfacial thermal resistance. J. Appl. Phys. 1997; 81,6692 .

[12] Osher S, Sethian JA. Fronts propagating with curvaturedependent speed: Algorithms based on Hamilton-Jacobi formulations. J. Comput. Phys. 1998;79(1):12-49.

[13] Pollack GL. Kapitza resistance. Rev. Mod. Phys. 1969;41:48.

[14] Stoner RJ, Maris HJ. Kapitza conductance and heatflow between solids at temperature from 50 to $300 \mathrm{~K}$. Phys. Rev. B. 1993;48:16373.

[15] Sukumar N, Chopp DL, Moës N, Belytschko T. Modeling holes and inclusions by level sets in the extended finite-element method. Comput. Meth. Appl. Mech. Eng. 2001;190:6183-6200.

[16] Swartz ET, Pohl RO. Thermal-resistance at interfaces. Appl. Phys. Lett. 1987;51:2200-2202.

[17] Torquato S, Rintoul MD. Effect of the interface on the properties of composite media. Phys. Rev. Lett. 1995;75:4067.

[18] Yvonnet J, He Q-C, Toulemonde C. Numerical modelling of the effective conductivities of composites with arbitrarily shaped inclusions and highly conducting interface. Compos. Sci. Technol. 2008;68:2828-2825. 